\title{
Safety and Efficacy of a Genetic Vaccine Targeting Telomerase Plus Chemotherapy for the Therapy of Canine B-Cell Lymphoma
}

\author{
Alessandra Gavazza, George Lubas, ${ }^{1}$ Arthur Fridman, ${ }^{2}$ Daniela Peruzzi, ${ }^{3}$ Joseph A. Impellizeri, ${ }^{4}$ \\ Laura Luberto, ${ }^{5,6}$ Emanuele Marra, ${ }^{6}$ Giuseppe Roscilli, ${ }^{6}$ Gennaro Ciliberto, ${ }^{7}$ and Luigi Aurisicchio ${ }^{6,8}$
}

\begin{abstract}
Client-owned pet dogs represent exceptional translational models for advancement of cancer research because they reflect the complex heterogeneity observed in human cancer. We have recently shown that a genetic vaccine targeting dog telomerase reverse transcriptase (dTERT) and based on adenovirus DNA electro-gene-transfer (Ad/DNA-EGT) technology can induce strong cell-mediated immune responses against this tumor antigen and increase overall survival of dogs affected by B-cell lymphosarcoma (LSA) in comparison with historical controls when combined with a cyclophosphamide, vincristine, and prednisone (COP) chemotherapy regimen. Here, we have conducted a double-arm clinical trial with an extended number of LSA patients, measured the antigenspecific immune response, and evaluated potential toxic effects of the immunotherapy along with a follow-up of patients survival for 3.5 years. The immune response was measured by enzyme-linked immunospot assay. The expression of dTERT was quantified by quantitative polymerase chain reaction. Changes in hematological parameters, local/systemic toxicity or organic dysfunction and fever were monitored over time during the treatment. dTERT-specific cell-mediated immune responses were induced in almost all treated animals. No adverse effects were observed in any dog patient that underwent treatment. The overall survival time of vaccine/COP-treated dogs was significantly increased over the COP-only cohort (>76.1 vs. 29.3 weeks, respectively, $p<0.0001)$. There was a significant association between dTERT expression levels in LSA cells and overall survival among vaccinated patients. In conclusion, Ad/DNA-EGT-based cancer vaccine against dTERT in combination with COP chemotherapy is safe and significantly prolongs the survival of LSA canine patients. These data confirm the therapeutic efficacy of dTERT vaccine and support the evaluation of this approach for other cancer types as well as the translation of this approach to human clinical trials.
\end{abstract}

\section{Introduction}

$\mathbf{R}$ ODENT CANCER MODELS ARE FUNDAMENTAL to studying the biology of pathways involved in cancer initiation, promotion, and progression. To date, several novel spontaneous murine cancer models have been generated that show heterogeneity, genomic instability, and an intact immune system. Nevertheless, they do not adequately represent several features that define cancer in humans, including environmental influence, the long periods of latency, the complex biology of cancer recurrence, the metastatic process, and outcomes to novel therapies (Ranieri et al., 2013). Among large animal models, nonhuman primates share size, gene expression, and an immune system similar to humans (Messaoudi et al., 2011). The high level of genetic homology with humans coupled with their outbred nature has made nonhuman primates invaluable preclinical models to assess the safety and efficacy of new drugs and biologics, such as antibodies (Finnefrock et al., 2009). Similarly, they are excellent models to evaluate vaccine platforms, especially for infectious diseases

\footnotetext{
${ }^{1}$ University of Pisa, Department of Veterinary Sciences, San Piero a Grado 56122, Pisa, Italy.

${ }^{2}$ Merck \& Co. Inc., West Point, PA 19486.

${ }^{3}$ Department of Medical-Surgical Sciences and Biotechnologies, "La Sapienza" University of Rome, 00185 Rome, Italy.

${ }^{4}$ Veterinary Specialty Center of the Hudson Valley, Wappingers Falls, NY 12590

${ }^{5}$ University of Catanzaro “Magna Graecia," Department of Experimental and Clinical Medicine Catanzaro, 88100 Italy.

${ }^{6}$ Takis, 00128 Rome, Italy.

${ }^{7}$ Istituto Nazionale Tumori "Fondazione Pascale," 80131 Naples, Italy.

${ }^{8}$ BIOGEM scarl, 83031 Ariano Irpino (AV), Italy.
} 
(Staprans et al., 2010) and more recently for cancer (Aurisicchio et al., 2007; Fattori et al, 2009; Dharmapuri et al, 2009) but are not suitable for efficacy studies in oncology.

In the pet population, with special reference to the dog, cancer is a spontaneous disease and dogs naturally develop cancers that share many characteristics with human malignancies. The compressed course of cancer progression in dogs allows timely assessment of new cancer therapies. Strong similarities to human cancers are observed including histological appearance, tumor genetics, environmental influence, biological behavior, and response to conventional therapies (Paoloni and Khanna, 2008). Examples include non-Hodgkin lymphoma (NHL), osteosarcoma, melanoma, prostate carcinoma, lung carcinoma, head and neck carcinoma, mammary carcinoma, and soft tissue sarcoma.

Malignant lymphosarcoma (LSA) is the most common hematopoietic malignancy in dogs, caused by clonal proliferation of lymphocytes in solid tissue with distinctive morphologic and immunophenotypic features. Among LSAs, NHL is the most common canine malignancy, accounting for up to $24 \%$ of all reported neoplasms. Similar to humans, the majority of canine NHL $(60 \%-80 \%)$ arises from malignant B cells. The median age of occurrence is about 7 years (Simon et al., 2008; Gavazza et al., 2009), and some studies have shown a positive association with exposure to herbicide and chemicals and with living in highly polluted areas (Hayes et al., 1991; Gavazza et al., 2001). Cytological evaluation of fine-needle aspirates is used to diagnose canine LSA, and the most frequent type is high-grade malignant polymorphic centroblastic based on updated Kiel classification (FournelFleury et al., 1997; Willmann et al., 2008; Gavazza et al., 2009). The most common presentation is a generalized lymphadenopathy corresponding to stage IV to $\mathrm{V}$ disease, with stage $\mathrm{V}$ describing tumor in blood, bone marrow, and other organ systems (Marconato, 2011). The current standard of care and cheapest treatment for canine B-lineage NHL is the combination chemotherapy regimen of cyclophosphamide, vincristine, and prednisolone (COP) or cyclophosphamide, vincristine, doxorubicin, and prednisone, which induces a temporary remission in approximately $85 \%$ of patients, but is rarely curative because the 2-year survival rate remains lower than 20\% (Marconato, 2011; Marconato et al., 2013). Treatment protocols mainly consist of a high-dose chemotherapy (induction phase) and a prolonged low-dose chemotherapy (maintenance phase). In particular, a 6- to 8-week induction with COP followed by a maintenance phase with prednisone and cyclophosphamide or chlorambucil and melphalan results in a median survival time of approximately 27 weeks (range 7-75; Gavazza et al., 2009; Marconato et al., 2013). More recently, short but dose-intense chemotherapy protocols (e.g., Madison, Wisconsin protocol) without maintenance provide disease-free intervals and survival times equivalent to protocols that include chronic maintenance therapy (Chun, 2009). Additionally, the use of half-body radiation to replace maintenance chemotherapy has demonstrated clinical efficacy and provides another option for maintaining durable remission times without the need for chronic chemotherapy (Chun, 2009; Lurie et al., 2009; Vail, 2010).

Telomerase is a ribonucleoprotein comprising an RNA component and a catalytic protein component (telomerase reverse transcriptase [TERT]; Meyerson et al., 1997; Nakamura et al., 1997). As reported for other species, telo- merase activity has been observed in the majority (>90\%) of canine tumors (Yazawa et al., 1999; Argyle and Nasir, 2003; Nasir et al., 2001; Nasir et al., 2008) contributing to maintenance of telomere length in cancer cells.

Genetic vaccines are emerging among the most promising methodologies. Accumulating evidence points to combinations of different genetic immunization modalities (heterologous prime/boost) as a powerful approach to induce superior immune responses and achieve greater clinical efficacy (Aurisicchio and Ciliberto, 2011a and b, 2012). We have recently shown that dTERT is expressed in B-cell LSA and that a genetic vaccine targeting this antigen and based on adenovirus (Ad) and DNA electro-gene-transfer (DNAEGT) was able to induce strong immune response in dogs affected by B-cell LSA. Most importantly, a standard chemotherapy regimen did not interfere with the effects of the immunotherapy, and survival of LSA dogs was significantly augmented in comparison to historical controls of chemotherapy-treated subjects (Peruzzi et al., 2010a).

The objective of the present study was to extend and further validate our observations in a double-arm trial of canine patients affected by B-cell LSA, and to evaluate the safety/toxicology profile and the impact on overall survival of this novel combined vaccine-chemotherapy treatment.

\section{Materials and Methods}

\section{Study design}

From May 2008 to October 2011, 108 client-owned dogs with LSA were evaluated and 42 were enrolled in the study and included in the vaccination/COP arm (VAC, 21 dogs) or COP arm (CTR, 21 dogs). The study was approved by Animal Ethical Committee of the University of Pisa and Ministry of Health. For each dog the veterinary staff of the Clinical Department performed a full initial clinical examination, completed the clinical record, and carried out the scheduled investigations to reach the diagnosis and clinical staging of LSA. At the first visit for the candidate dogs, peripheral blood, lymph node, and bone marrow samples were collected, and thoracic and abdomen X-rays and abdomen ultrasound were performed. An informed consent was distributed and approved by owners. Cytological smears and immunophenotyping were performed to classify the LSA by fine-needle aspiration of lymph nodes and bone marrow aspirate.

\section{Blood tests}

Complete blood count, serum biochemical profile of at least 10-12 analytes (including total protein, albumin, urea, creatinine, alanine aminotransferase, aspartate aminotransferase, alkaline phosphatase, gamma-glutamyltransferase, calcium, phosphorus, iron, cholesterol, glucose, sodium, potassium), coagulation profile (prothrombin time, activated partial thromboplastin time, fibrinogen), serum protein electrophoresis, urinalysis, and serological titers using immunofluorescence antibody testing for Leishmania infantum, Ehrlichia canis, and Rickettsia rickettsii were performed from peripheral blood.

\section{Immunization of dogs}

The genetic components of the dTERT vaccine (Ad6-dTERT ${ }_{\text {opt }}$ and pV1J-dTERT.LTB ${ }_{\text {opt }}$ ) have been described elsewhere (Peruzzi

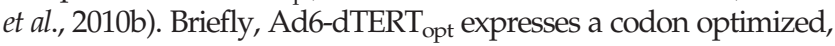


catalytically inactive (D702A, V703I) full-length canine telomerase, and pV1J-dTERT.LTB ${ }_{\text {opt }}$ encodes the same cDNA fused at the N-terminal with a tissue plasminogen activator leader sequence and at the C-terminal with the codon-optimized B subunit of Escherichia coli heat-labile enterotoxin (LTB). For Ad vaccination, dogs were injected in femoral biceps muscle with a dose of $10^{11}$ Ad viral particles (vp). The DNA injection consisted of a 1-mL solution (split over two injection sites with $0.5 \mathrm{~mL} /$ site) containing $5 \mathrm{mg}$ of pV1J-dTERT.LTB ${ }_{\text {opt }}$ in dogs' tibial muscles. Electrical conditions for electroporation consisted of 8 pulse trains of 100 square bipolar wave pulses ( $1 \mathrm{sec}$ each) delivered every other second for a total treatment time of $10 \mathrm{sec}$. The pulse length was $2 \mathrm{msec} /$ phase with a frequency and amplitude of $100 \mathrm{~Hz}$ and $100 \mathrm{~mA}$, respectively (constant current mode). Dogs were anesthetized with medetomidine at about $10 \mu \mathrm{g} / \mathrm{kg}$ (Domitor, Eli Lilly, Italia Spa, Sesto Fiorentino, Italy) intravenously (IV) and with propofol at about $5 \mathrm{mg} / \mathrm{kg}$ (Rapinovet, Intervet Italia srl, Peschiera Borromeo, Italy) IV to carry out the electroporation. To reverse the anesthesia, atipamezole at about $10 \mu \mathrm{g} / \mathrm{kg}$ (Antisedan, Eli Lilly, Italia Spa, Sesto Fiorentino, Italy) intramuscularly was used. Dogs with tumors enrolled in the vaccination protocol received two Ad6$\mathrm{dTERT}_{\text {opt }}$ injections followed by one or more cycles, each composed by $3 \times$ DNA-EGT. Blood was collected at indicated time points, and peripheral mononuclear cells (PBMCs) were isolated by density gradient centrifugation columns (Accuspin, Sigma, St. Louis, MO). Fresh or frozen PBMC samples were analyzed for immunologic assays.

\section{Peptides}

Lyophilized dTERT peptides were purchased from JPT Peptide Technologies GmbH (Berlin, Germany) and resuspended in dimethyl sulfoxide at $40 \mathrm{mg} / \mathrm{mL}$. Pools of 15 amino acid peptides overlapping by 11 residues were assembled as described previously (Peruzzi et al., 2010b). Four pools of 70 peptides, respectively, were formed for the whole length of the TERT protein. The final concentration of each peptide in the pools was $0.57 \mathrm{mg} / \mathrm{mL}$.

\section{Interferon- $\gamma$ enzyme-linked immunospot assay}

The enzyme-linked immunospot (ELISPOT) assay was performed using a standard kit for the detection of canine interferon (IFN) $\gamma$ (RnD Systems) according to the manufacturer's instructions. Purified dog PBMCs (Accuspin system, Sigma, St. Louis, MO) were plated in duplicate in 96-well MAIP plates (Millipore, Temecula, CA) coated overnight with an anti-dog IFN $\gamma$ antibody, at a density of $4 \times 10^{5}$ and $2 \times 10^{5}$ cells / well. Cells were incubated for $20 \mathrm{hr}$ at $37^{\circ} \mathrm{C}$ with $2 \mu \mathrm{g} / \mathrm{mL}$ suspension of each peptide. Concanavalin A was used as positive internal control at $10 \mu \mathrm{g} / \mathrm{mL}$. Plates were washed and incubated for $12 \mathrm{hr}$ at $4^{\circ} \mathrm{C}$ with biotinconjugated anti-dog IFN $\gamma$ (50 $\mu \mathrm{g} /$ well). Spots were detected after incubation with streptavidin-AP followed by 5-bromo4-chloro-3-indoly-phosphate/nitro blue tetrazolium (NBT/ BCIP) substrate (50 $\mu \mathrm{L} /$ well; Pierce Biotechnology, Rockford, IL). Plates were washed and air-dried, and spots were counted with an automated ELISPOT reader. In most cases, the assay was performed with fresh PBMCs. When this was not possible, PBMCs were frozen according to standard procedure and analyzed at a later time point.

\section{Real-time quantitative polymerase chain reaction}

Fine-needle biopsies were obtained from patients diagnosed with B-cell LSA, and stored in RNAlater (Qiagen). Total RNA was isolated with the Qiagen RNeasy Mini Reagent Set (Qiagen Venlo, The Netherlands), according to the manufacturer's instructions. Real-time quantitative polymerase chain reaction (PCR) was performed as previously described (Peruzzi et al., 2010a). Briefly, $1 \mu \mathrm{g}$ of total RNA was used to perform reverse transcription of RNA and real-time PCR (TaqMan) assay using primer/probe set (Cf02671898_g1) that recognized canine TERT (Applied Biosystems, Life Technologies Italia, Monza, Italy) according to manufacturer's instructions. The reactions were performed using a TaqMan Gold reverse transcription (RT)-PCR kit (PE Applied Biosystems) and included a 30-min reverse transcription step at $50^{\circ} \mathrm{C}$ followed by $10 \mathrm{~min}$ at $95^{\circ} \mathrm{C}$ and 40 cycles of amplification using the universal TaqMan standardized conditions $\left(15-\mathrm{sec}, 95^{\circ} \mathrm{C}\right.$ denaturation step followed by a 1-min, $60^{\circ} \mathrm{C}$ annealing/extension step). RNA transcribed from a plasmid containing dTERT (Peruzzi et al., 2010a) was used as a standard to establish genome equivalents. All reactions were run in duplicate by using the ABI Prism 7900 sequence detection system (Applied Biosystems).

\section{Statistical analysis}

Student's $t$-test was performed where indicated. Log-rank test was used to evaluate the difference between progressionfree survival and overall survival (OS) between groups. Spearman's rank correlation was used to test for an association between dTERT expression levels and OS; $p$-values $<0.05$ were considered statistically significant. Statistical analyses were performed using Matlab 7.11 (The MathWorks, Natick, MA).

\section{Results}

\section{Study design and patients}

A total of 21 stage III-IV dogs for vaccine/COP (VAC) and 21 for COP (CTR) arm possessed the requirements for the clinical trial and were enrolled (Tables 1 and 2, respectively). Dogs were assigned to the two treatment arms based on their owners' consent. The VAC group included 61.9\% males, $23.8 \%$ females, and $14.3 \%$ neutered females. Breeds included Labrador and Golden Retrievers (3), Doberman (2), German Shepherd (3), Bernese mountain (2), Shitzu (1), Rottweiler (2), Dogo Argentino (1), English Setter (2), and mixed-breed dogs (5). Similarly, the CTR group comprised $47.6 \%$ of males, $42.9 \%$ females, and 9.5\% neutered females. Breeds included German shepherd (6), Bull Mastiff (2), Rottweiler (2), English Setter (1), Fox Terrier (1), Bull Terrier (1), Doberman (1), Boxer (1), Great Dane (1), Beagle (1), and mixed-breed dogs (4). The median age was 7 years (range, 5-11 years) and 8 years (range 4-13 years) for the VAC and CTR groups, respectively. Fifteen and 14 dogs of the VAC and CTR arms, respectively, received one or more treatments before the induction chemotherapy, such as steroids (57\% and $28.5 \%$ ) and antibiotics (most cases). All subjects were affected by high-grade type LSA, B immunophenotype and were grouped as follows:

- VAC arm: centroblastic polymorphic (14 cases, 66.7\%), centro-immunoblastic (4, 19.0\%), immunoblastic (2, $28.6 \%)$, and lymphoblastic $(1,14.3 \%)$ 
Table 1. VAC Cohort: Vaccine/COP-Treated Subjects

\begin{tabular}{|c|c|c|c|c|c|c|c|}
\hline Code & $\begin{array}{l}\text { Age } \\
\text { (years) }\end{array}$ & Breed/sex & Classification & $\begin{array}{l}\text { Use of } \\
\text { steroids }\end{array}$ & Treatments recorded before chemo/immunotherapy & $\begin{array}{l}\text { EGT } \\
\text { cycles }\end{array}$ & $\begin{array}{l}\text { Use } \\
\text { of dox }\end{array}$ \\
\hline VAC 1 & 6 & $\begin{array}{l}\text { Doberman } \\
\text { Pinscher, } \mathrm{m}\end{array}$ & Immunoblastic & Yes & $\begin{array}{l}\text { PO: prednisone, fenbendazole, clindamycin, } \\
\text { amoxicillin-clavulanic acid; SC: cefotaxime }\end{array}$ & 2 & Yes \\
\hline VAC 2 & 7 & Dogo Argentino, fs & Centroblastic & No & None & 2 & No \\
\hline VAC 3 & 5 & German Shepherd, $\mathrm{m}$ & Immunoblastic & Yes & $\begin{array}{l}\text { SC: ketoprofen, enrofloxacin, } \\
\text { methylprednisolone }\end{array}$ & 2 & Yes \\
\hline VAC 4 & 7 & Mixed, $\mathrm{m}$ & Centroblastic & No & $\begin{array}{l}\text { PO: chondroitin sulfate-glucosamine- } \\
\text { quercetin, carprofen }\end{array}$ & 2 & No \\
\hline VAC 5 & 9 & Mixed, m & $\begin{array}{l}\text { Centroblastic/ } \\
\text { immunoblastic }\end{array}$ & Yes & $\begin{array}{l}\text { IV: vincristine; PO: prednisone, } \\
\text { cyclophosphamide }\end{array}$ & 3 & No \\
\hline VAC 6 & 9 & Mixed, $\mathrm{m}$ & Centroblastic & No & None & 2 & Yes \\
\hline VAC 7 & 6 & $\begin{array}{l}\text { Bernese Mountain } \\
\text { dog, fs }\end{array}$ & $\begin{array}{l}\text { Centroblastic/ } \\
\text { immunoblastic }\end{array}$ & Yes & $\begin{array}{l}\text { Ophthalmic suspension: tobramycin- } \\
\text { dexamethasone }\end{array}$ & 2 & Yes \\
\hline VAC 8 & 6 & $\begin{array}{l}\text { Bernese Mountain } \\
\text { dog, } \mathrm{m}\end{array}$ & Lymphoblastic & Yes & SC: ceftriaxone; PO: doxycycline, prednisone & 2 & Yes \\
\hline VAC 9 & 10 & Shih-Tzu f & Centroblastic & Yes & PO: amoxicilline-clavulanic acid, prednisone & 0 & Yes \\
\hline VAC 10 & 9 & German Shepherd, m & Centroblastic & Yes & PO: metronidazole-spiramycin, prednisone & 2 & Yes \\
\hline VAC 11 & 9 & Mixed, $\mathrm{m}$ & Centroblastic & No & None & 2 & Yes \\
\hline VAC 12 & 10 & Labrador retriever $\mathrm{f}$ & Centroblastic & No & None & 2 & Yes \\
\hline VAC 13 & 8 & English Setter, f & $\begin{array}{l}\text { Centroblastic/ } \\
\text { immunoblastic }\end{array}$ & Yes & $\begin{array}{l}\text { PO: amoxicillin-clavulanic acid, prednisone, } \\
\text { dexamethasone }\end{array}$ & 0 & Yes \\
\hline VAC 14 & 10 & English setter, $\mathrm{f}$ & Centroblastic & Yes & PO: amoxicillin-clavulanic acid, prednisone & 1 & No \\
\hline VAC 15 & 7 & German Shepherd, $\mathrm{m}$ & $\begin{array}{l}\text { Centroblastic/ } \\
\text { immunoblastic }\end{array}$ & Yes & SC: ceftriaxone; PO: allopurinol, prednisone & 2 & Yes \\
\hline VAC 16 & 5 & Rottweiler, m & Centroblastic & No & $\begin{array}{l}\text { PO: doxycycline, B-complex vitamins, } \\
\text { aluminum and magnesium hydroxide }\end{array}$ & 1 & Yes \\
\hline VAC 17 & 5 & Labrador retriever, $\mathrm{m}$ & Centroblastic & No & None & 3 & No \\
\hline VAC 18 & 6 & Rottweiler, m & Centroblastic & No & None: venous port access in-site & 1 & Yes \\
\hline VAC 19 & 4 & $\begin{array}{l}\text { Doberman } \\
\text { Pinscher, fs }\end{array}$ & Centroblastic & Yes & PO: amoxicillin-clavulanic acid, prednisone & 2 & Yes \\
\hline VAC 20 & 11 & Mixed, m & Centroblastic & No & oxicillin-clavulanic acid & 2 & No \\
\hline VAC 21 & 8 & Golden retriever, $\mathrm{f}$ & Centroblastic & Yes & PO: prednisone & 2 & Yes \\
\hline
\end{tabular}

VAC, vaccination/COP arm; COP, cyclophosphamide, vincristine, and prednisone; $\mathrm{m}$, male; $\mathrm{f}$, female, fs, female neutered; $\mathrm{PO}$, per os; $\mathrm{SC}$, subcutaneous; IV, intravenous; dox, doxorubicin; EGT, electro-gene-transfer.

- CTR arm: centroblastic polymorphic (18 cases, 85.7\%), centroblastic/centrocytic $(1,14.3 \%)$, pleomorphic $(1,14.3 \%)$, and lymphoblastic $(1,14.3 \%)$

The lymph node involvement was generalized with different prevalent locations. Selected animals did not show any additional significant disease. All dogs included in the study received a standard chemotherapy induction protocol (induction phase [IP]) with vincristine $\left(0.75 \mathrm{mg} / \mathrm{m}^{2}\right.$ IV once a week), cyclophosphamide (50 mg/ $\mathrm{m}^{2}$ orally $3-4$ days/week), and prednisone $\left(40 \mathrm{mg} / \mathrm{m}^{2}\right.$ orally daily for the first week, then $20 \mathrm{mg} / \mathrm{m}^{2}$ orally daily for the further weeks) for 6-8 weeks. Only dogs that achieved complete clinical remission (CR) after 6-8 weeks of treatment were enrolled. At CR, dogs received a maintenance protocol (maintenance phase [MP]) therapy with chlorambucil $\left(2 \mathrm{mg} / \mathrm{m}^{2}\right.$ orally $2-3$ days/week), melphalan ( $2 \mathrm{mg} / \mathrm{m}^{2}$ orally $2-3$ days/week), and vincristine (0.60$0.70 \mathrm{mg} / \mathrm{m}^{2}$ IV every $3-4$ weeks). In several cases, dogs were treated with doxorubicin $\left(30 \mathrm{mg} / \mathrm{m}^{2}\right.$, every 3 weeks) as a consequence of relapse of the disease in addition to the maintenance treatment (see Tables 1 and 2).

Two weeks after CR, the VAC group was treated with dTERT genetic vaccine along with maintenance chemotherapy. The vaccine regimen consisted of two injections of Ad $\left(10^{11} \mathrm{vp}\right)$ in the femoral biceps at 2-week intervals followed by one or more cycles of three DNA-EGT ( $5 \mathrm{mg}$ /injection) every 2 weeks in the tibial muscle (see Fig. 1), as previously described (Peruzzi et al., 2010a). Most of the dogs received the complete treatment consisting of two Ad and two DNAEGT cycles (Table 1). Two cases (VAC5 and VAC17) received a third DNA-EGT cycle, while two (VAC 9 and VAC13) were not boosted with DNA-EGT due to severe disease relapse and withdrawal on the owner's decision, respectively.

\section{T-cell immune response}

Immune response against tumor-associated antigens may occur naturally in cancer patients, irrespective of immune therapy. To verify whether a spontaneous T-cell immune response is detectable in LSA patients after chemotherapy and to define a background reactivity against dTERT peptides, PBMCs were collected either from untreated, healthy dogs and LSA patients at CR (the VAC arm), 2-3 weeks after the IP. An ELISPOT assay was performed as described in the Materials and Methods section. As shown in Figure 2, the background of the assay was low $(9.12 \pm 14.46$ Spot-forming cells [SFCs] $/ 10^{6}$ PBMCs for LSA patients), and no significant difference in the immune response was detected between healthy and LSA groups. In two cases the signal was around 50 SFCs $/ 10^{6}$ PBMCs. These data indicate that low $/$ no 
Table 2. CTR Cohort: COP-Treated Subjects

\begin{tabular}{|c|c|c|c|c|c|c|}
\hline Code & $\begin{array}{l}\text { Age } \\
\text { (years) }\end{array}$ & Breed/sex & Classification & $\begin{array}{l}\text { Use of } \\
\text { steroids }\end{array}$ & Treatments recorded before chemotherapy & $\begin{array}{l}\text { Use } \\
\text { of dox }\end{array}$ \\
\hline CTR 1 & 5 & German Shepherd, f & Centroblastic & No & SC: ceftriaxone; PO: doxycycline & Yes \\
\hline CTR 2 & 5 & Mixed, m & Centroblastic & Yes & PO: doxycycline, prednisone, aspirin & Yes \\
\hline CTR 3 & 6 & Bull Mastiff, $\mathrm{f}$ & Centroblastic & No & PO: clindamycin & Yes \\
\hline CTR 4 & 4 & Bull Mastiff, m & Centroblastic & No & $\begin{array}{l}\text { Gentamicin + betamethasone } \\
\text { cutaneous ointment }\end{array}$ & Yes \\
\hline CTR 5 & 12 & Mixed, f & $\begin{array}{l}\text { Centroblastic/ } \\
\text { centrocytic }\end{array}$ & No & PO: enalapril & Yes \\
\hline CTR 6 & 7 & English Setter, $\mathrm{m}$ & Pleomorphic & No & None & Yes \\
\hline CTR 7 & 12 & German Shepherd, $\mathrm{m}$ & Centroblastic & Yes & PO: prednisone & Yes \\
\hline CTR 8 & 1 & German Shepherd, f & Centroblastic & No & None & Yes \\
\hline CTR 9 & 8 & Fox Terrier, $\mathrm{f}$ & Centroblastic & No & $\begin{array}{l}\text { PO: metronidazole-spiramycin, } \\
\text { furosemide }\end{array}$ & Yes \\
\hline CTR 10 & 8 & Bull Terrier, m & Centroblastic & Yes & SC: rifamycin, methylprednisolone & Yes \\
\hline CTR 11 & 13 & Mixed, m & Centroblastic & No & None & No \\
\hline CTR 12 & 9 & German Shepherd, m & Centroblastic & No & None & No \\
\hline CTR 13 & 9 & German Shepherd, f & Centroblastic & Yes & $\begin{array}{l}\text { PO: amoxicillin-clavulanic acid, } \\
\text { prednisone, ranitidine, } \\
\text { S-adenosyl methionine }\end{array}$ & No \\
\hline CTR 14 & 4 & Rottweiler, f & Lymphoblastic & Yes & PO: doxycycline, prednisone & Yes \\
\hline CTR 15 & 10 & Doberman Pinscher, $f$ & Centroblastic & No & PO: metronidazole & Yes \\
\hline CTR 16 & 8 & Rottweiler, m & Centroblastic & No & None & Yes \\
\hline CTR 17 & 8 & German Shepherd, fs & Centroblastic & No & PO: enrofloxacin & No \\
\hline CTR 18 & 13 & Mixed, fs & Centroblastic & No & None & No \\
\hline CTR 19 & 8 & Boxer, $\mathrm{f}$ & Centroblastic & No & None & Yes \\
\hline CTR 20 & 7 & Great Dane, m & Centroblastic & Yes & PO: amoxicillin-clavulanic acid, prednisone & Yes \\
\hline CTR 21 & 7 & Beagle, $\mathrm{m}$ & Centroblastic & No & PO: amoxicillin-clavulanic acid, enalapril & Yes \\
\hline
\end{tabular}

CTR, COP arm.

spontaneous immune responses against dTERT are present in this cancer population after the IP.

We have previously shown that most LSA dogs mount a potent cell-mediated immune response against the target tumor antigen upon treatment with dTERT genetic vaccine and that this can be boosted over time by repeated DNAEGT (Peruzzi et al., 2010a, 2010b). PBMCs from the VAC arm were collected at $C R$ (before vaccination), 2 weeks after the second Ad vaccination, and 2-3 weeks after the DNA-EGT cycle (see Fig. 1). Additional blood collections were performed after the second DNA-EGT cycle. PBMCs from four dogs from the CTR arm (CTR15, 18, 20, 21) and obtained at comparable time points after the IP and during the MP were also analyzed. The number of T cells specific for dTERT was enumerated by ELISPOT. Nineteen out of 21 VAC dogs $(90.5 \%)$ showed a strong cell-mediated immune response against the protein component of dog telomerase (Fig. 2B). For technical reasons it was not possible to determine the immune response for VAC8 and VAC13 patients. The cellmediated immune response ranged from 7 to 663 SFCs/ million (average, 223 \pm 168 ) PBMCs after Ad injections with maximal peak from 82 to 1464 SFCs/million (average: $445 \pm 330)$ PBMCs after DNA-EGT boosts. In seven cases (VAC 2, 3, 9, 11, 14, 19, 21) the cell-mediated immune response peak was reached immediately after the two Ad injections. No significant spontaneous immune response was measured in the PBMCs obtained from the four available CTR cases during standard chemotherapy (Fig. 2B).

To further characterize the T-cell immune response reactivity and provide information on whether particular regions of dTERT protein component are more immunogenic within each dog among vaccinated dogs in the VAC cohort, we performed an ELISPOT assay using overlapping peptide pools (dTert A, B, C, D), which sequentially covered the protein from the $\mathrm{N}$-terminal to the $\mathrm{C}$-terminal. The analysis was specifically carried out on PBMCs drawn after the Ad/ DNA-EGT cycle 1 vaccination. Figure $2 \mathrm{C}$ shows that six dogs mounted a multi-epitope response distributed over all dTert peptide pools (VAC4, 5, 6, 11, 15, 17), five reacted to two or three pools (VAC2, 10, 18, 20, 21), and five had a dominant specificity for single pool A or B (VAC1, 3, 7, 12, 19). The specificity of the response was maintained also for the following time points (data not shown).

Taken together, these data further demonstrate the strong immunogenicity of dTERT genetic vaccine and show that the type and quality of elicited T-cell immune response is patient specific.

\section{dTERT vaccine does not show significant side effects}

To assess potential side effects connected with the immunotherapy, body weight and temperature were measured throughout the entire course of the study. No significant changes were noticed during the entire course of the study (not shown). To monitor signs of toxicity and/or to detect indications of autoimmunity, vaccinated animals were constantly monitored for abnormal values in hematological blood parameters. In fact, TERT is expressed in actively replicating cells and TERT-specific T-lymphocyte passive transfer in a mouse system has shown partial depletion of normal B cells, which also express telomerase (Ugel et al., 2010). The hematologic parameters were measured before 


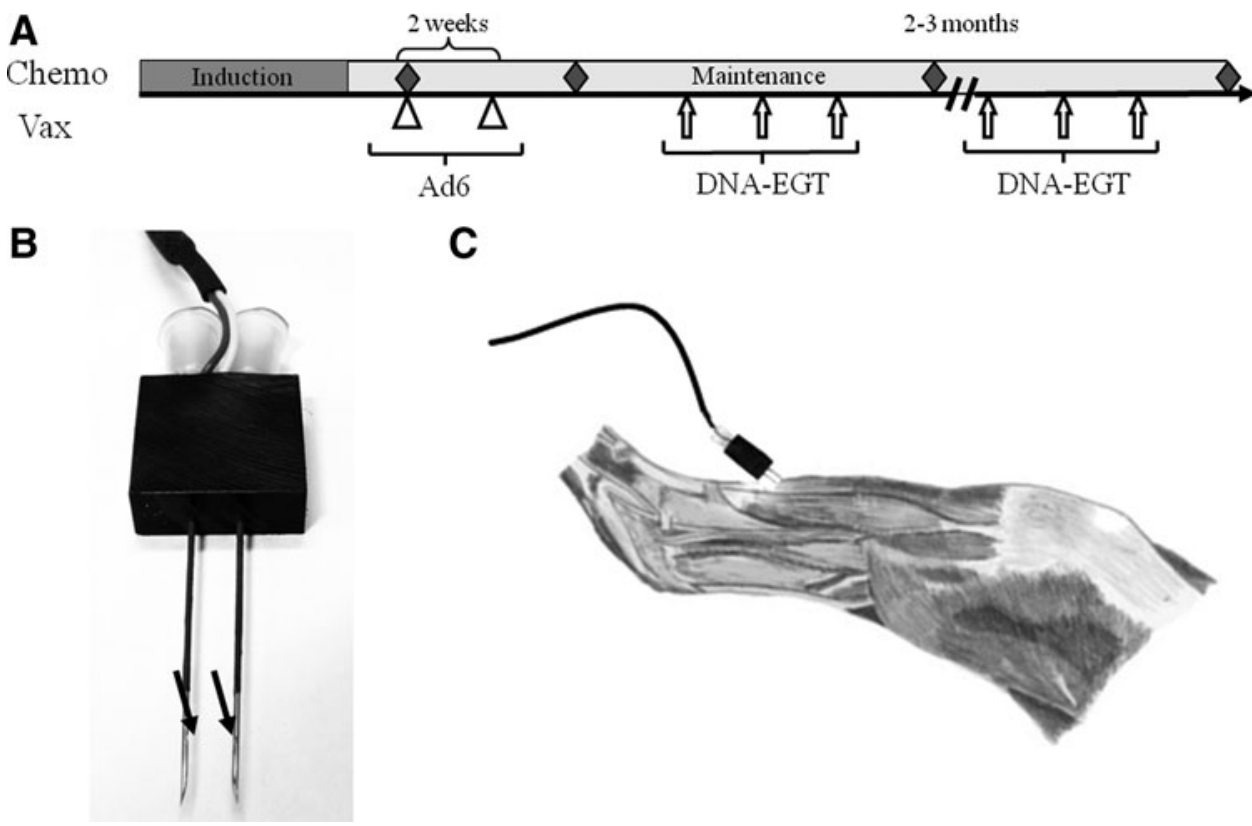

FIG. 1. Vaccination schedule and electro-gene-transfer (EGT) procedure. (A) Schematic representation of the vaccination and chemotherapy combination schedule. Dark and light gray indicate the induction and maintenance phases of chemotherapy. Two weeks after the end of induction phase (week 8-10) and during the maintenance phase, dogs were vaccinated with adenovirus (Ad; triangles, 2 weeks apart) and 4-6 weeks later with DNA-EGT cycles (arrows) consisting of three injections spaced by 2-week intervals. A second cycle was repeated 2-3 months later. Black diamonds show the time points when blood was collected for detection of the immune response and other analyses. (B) Needles and electrodes. The side-hole needles were modified to inject the DNA solution in the area between them and act as electrodes, since they are connected with the pulse generator. Arrows indicate the side holes. (C) Schematic representation of the DNA injection in the tibial muscle and the DNA-EGT procedure. Needles were inserted into the muscle at an angle of approximately $45^{\circ}$.

(PRE, at diagnosis) and during chemotherapy or chemotherapy plus immunotherapy (post Ad/IP: week 6 after completion of the IP; post DNA-EGT or during MP: week 14-18 after completion of the IP). There were variations over time due to COP therapy, which were observed in both cohorts. Some of them are shown in Supplementary Table S1 (Supplementary Data are available online at www.liebertpub .com/hum). No effects on white blood cells counts were observed over time, while red blood cells were significantly reduced after the IP but recovered during the MP. The same trend was observed for hemoglobin and hematocrit (not shown). On the other hand, red cell distribution width and platelet counts increased upon treatment in both groups (Supplementary Table S1). Therefore, no significant hematological side effects connected with the immunizations were detectable in treated animals.
In conclusion, neither significant side effects nor apparent phenomena of autoimmunity were observed in relation with the genetic vaccine and the immune response against dTERT.

\section{VAC arm shows an increased overall survival compared to CTR arm associated with dTERT expression}

Animals in the VAC and CTR arms were monitored over time for relapse of the disease and survival. Figure 3 shows the time to first relapse and OS of the VAC and CTR patient groups. Most disease relapses during maintenance chemotherapy occurred within the 15 weeks after chemotherapy initiation. No significant increase in this parameter ( $p>0.1$; exact log-rank test) was observed between the two cohorts (median, VAC and CTR arms, 11.4 and 11.3 weeks,

FIG. 2. Characterization of T-cell immune response. (A) Spontaneous immune response against dog telomerase reverse transcriptase (dTERT). Peripheral mononuclear cells (PBMCs) were purified from six healthy dogs (three beagles, one Labrador Retriever, and two mixed) and the 21 lymphosarcoma (LSA) dogs from the VAC cohort after the induction phase (IP). Each single dot indicates the total reactivity to dTERT peptide pools of a single subject. The bars show the geometric mean of the groups: $5.12 \pm 6.88$ and $9.12 \pm 14.46$ for healthy and LSA dogs, respectively. Student's $t$-test, $p=0.35$. (B) Kinetics of the dTERT-specific T-cell response in VAC arm. LSA dogs were treated as described in the text and indicated in the top scheme in Fig. 1. PBMCs were purified at the indicated time points and the response was measured by enzyme-linked immunospot (ELISPOT) with dTERT peptide pools. The graph is in log scale. *Student's $t$-test, $p<1 \times 10^{5}$ compared with prevaccination. The total reactivity for dTERT peptide pools is calculated as Total reactivity $=\Sigma(\mathrm{dTert} A+\mathrm{dTertB}+\mathrm{dTertC}+\mathrm{dTertD}$ pools) -4 (dimethyl sulfoxide). (C) Distribution of the immune reactivity within dTERT. dTertA, dTertB, dTertC, and dTertD pools were used to stimulate PBMCs 2 weeks after the second Ad injection. ELISPOT assays were run in quadruplicates at the indicated time points. 
A

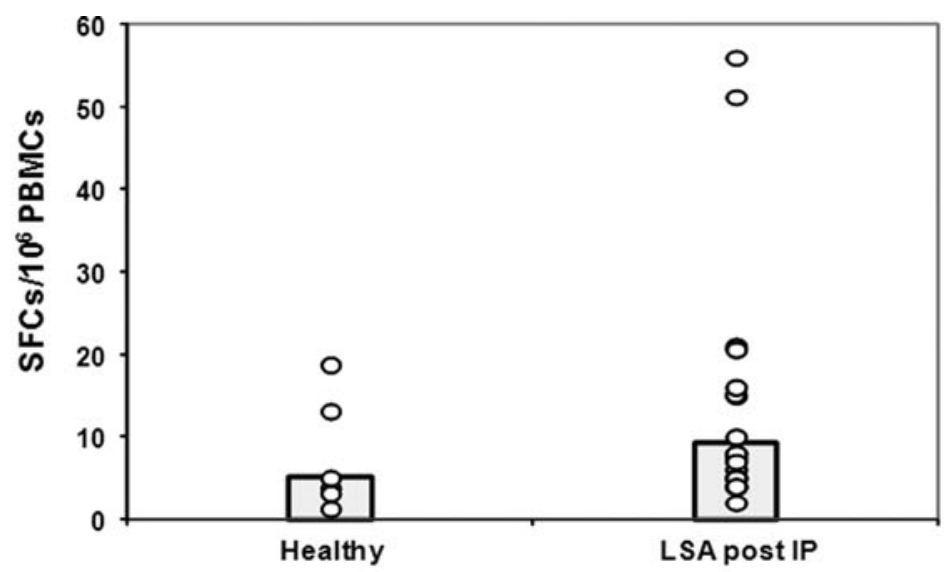

B 10000 VAC Arm

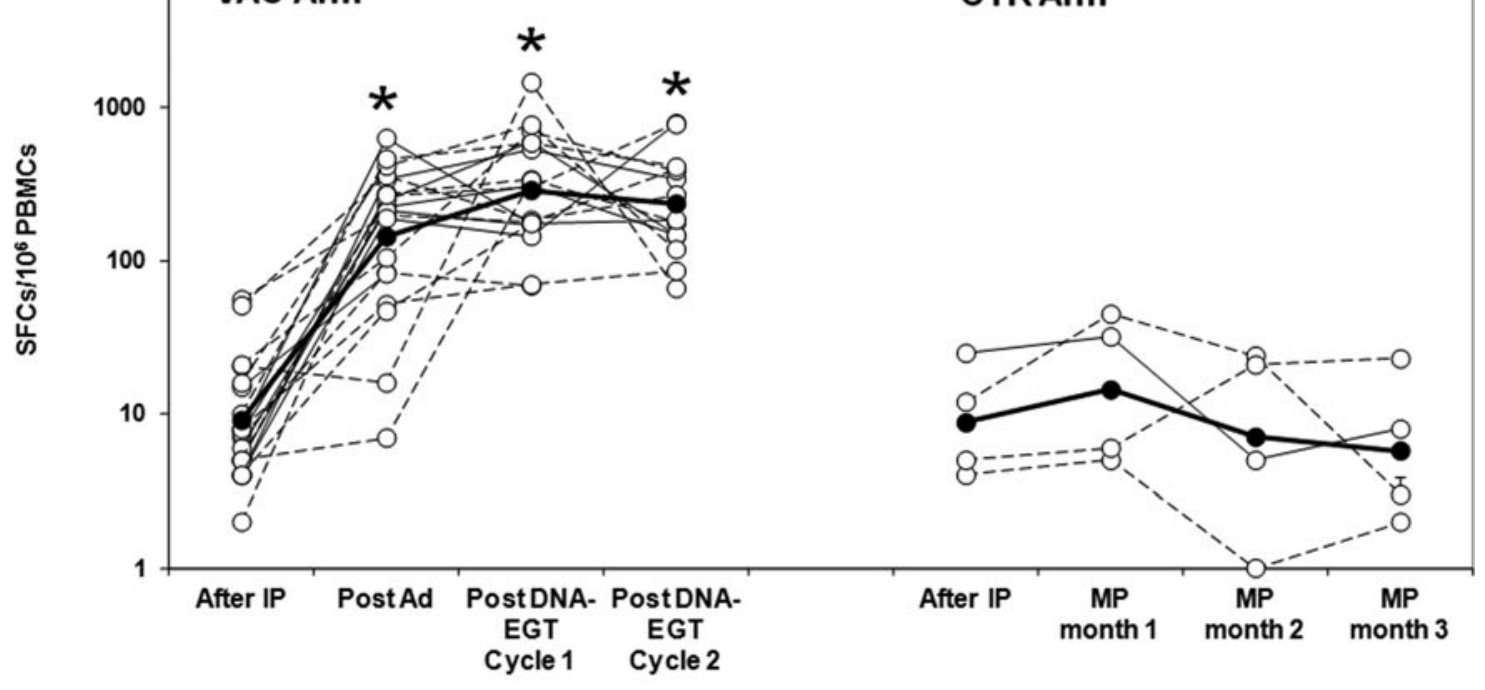

C

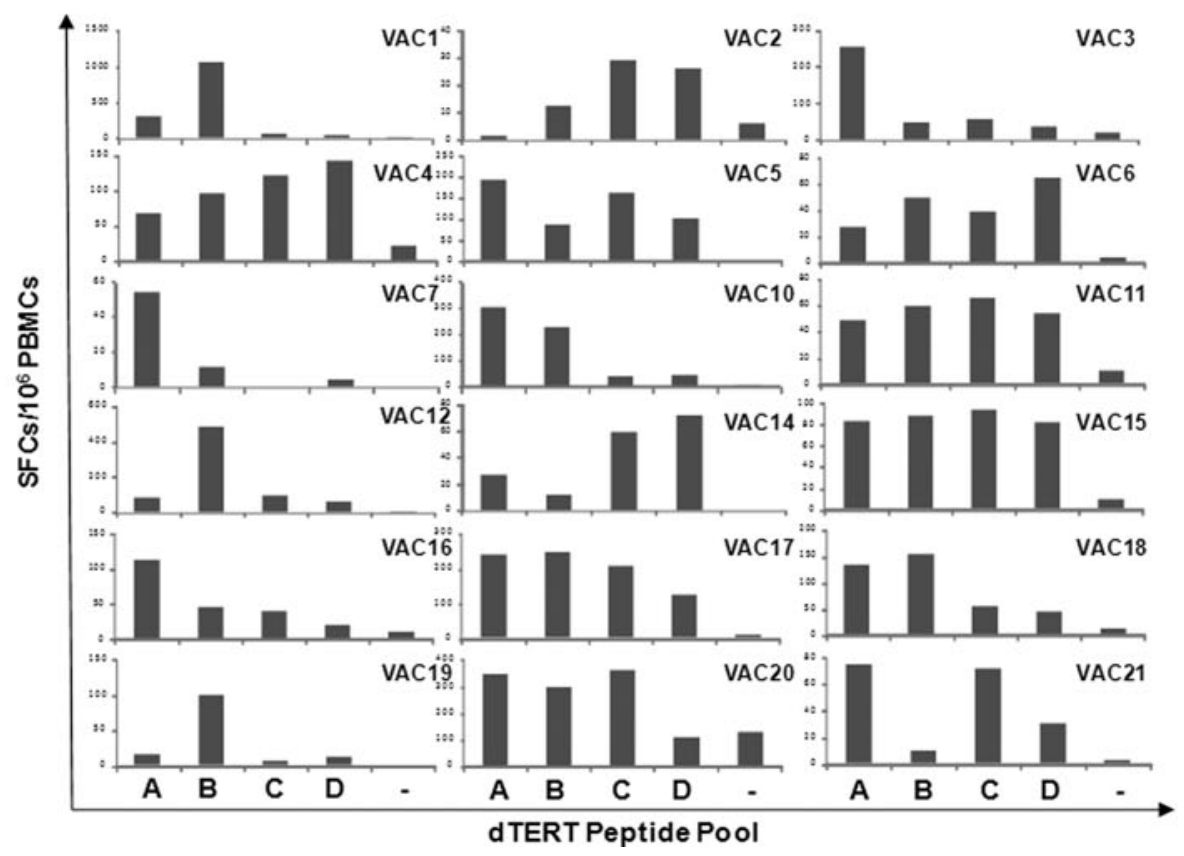



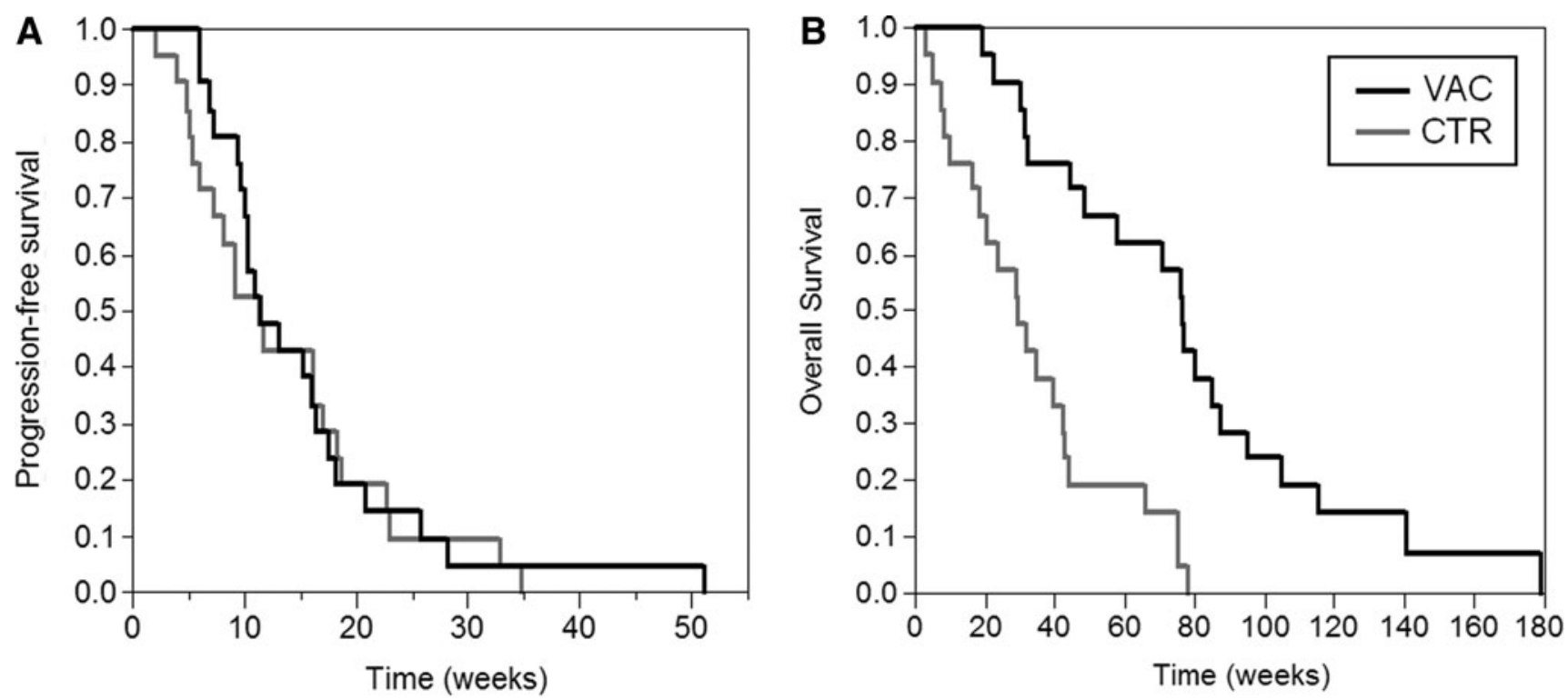

FIG. 3. dTERT vaccine augments LSA patients' survival. (A) Kaplan-Meier plot of time to first relapse. Vaccinated dogs (VAC, black line; $n=21)$ do not show a significantly longer progression-free survival than dogs on chemotherapy alone (CTR, gray line; $n=21)$. Median progression-free survival is 11.4 and 11.3 weeks for VAC and CTR groups, respectively $(p>0.1, \log -$ rank test). (B) Kaplan-Meier plot of overall survival. VAC arm (black line; $n=21$ ) show a significantly longer overall survival than CTR arm (gray line; $n=21$ ). Median overall survival (OS) is 76.1 and 29.3 weeks for VAC and CTR cohort, respectively $(p<0.0001, \log$-rank test).

respectively, Fig. 3A). Nevertheless, a significant increase in OS was observed for the VAC over the CTR group $(p<0.0001$, exact log-rank test). The median survival was 76.1 vs. 29.3 weeks for VAC and CTR arm, respectively (Fig. 3B). There was no observable difference in survival both by sex and age. The evaluations included all dogs initiating treatment, irrespective of reason for not completing the study (i.e., VAC9 and 13). No correlation between the extent of T-cell immune response and the survival was observed (not shown).

To assess whether dTERT expression may represent a prognostic factor for survival, RNA was extracted from six lymph node fine needle aspirates from dogs of the VAC arm (VAC1, 3, 10, 11, 12, 14). As previously observed (Peruzzi et al., 2010a), dTERT mRNA was detected by real-time PCR in all samples at different levels. Interestingly, there was a statistically significant association between dTERT expression levels and OS among the six available vaccinated patients (Fig. 4).

These data further confirm that dTERT genetic vaccine has a positive impact on LSA dogs, when combined with COP therapy, and suggest that the levels of dTERT expression may represent a prognostic factor to predict dTERT vaccine clinical efficacy.

\section{Discussion}

LSA is the most common hematopoietic tumor in dogs, and about $80 \%$ of cases are B types (Willmann et al., 2009). It is very aggressive, and no cure is available apart from chemotherapy. Dogs that undergo standard COP usually survive for 8 months on average even if they often relapse (Gavazza et al., 2009). Recent reports show that canine LSA is treatable with experimental immunotherapy approaches in addition to standard chemotherapy, such as adoptive cell therapy (O'Connor et al., 2012) and tumor RNA-loaded, CD40-activated B cells (Sorenmo et al., 2011). However, these personalized cell therapy agents are cumbersome to produce and generally very expensive. For these reasons, alternative technologies that combine lower manufacturing costs and a more standardized production process with the induction of strong immunological responses against tumor antigen(s) are needed. In this respect, a promising avenue is represented by gene-based vaccines. In our previous study (Peruzzi et al., 2010a), we showed that dTERT genetic vaccine was

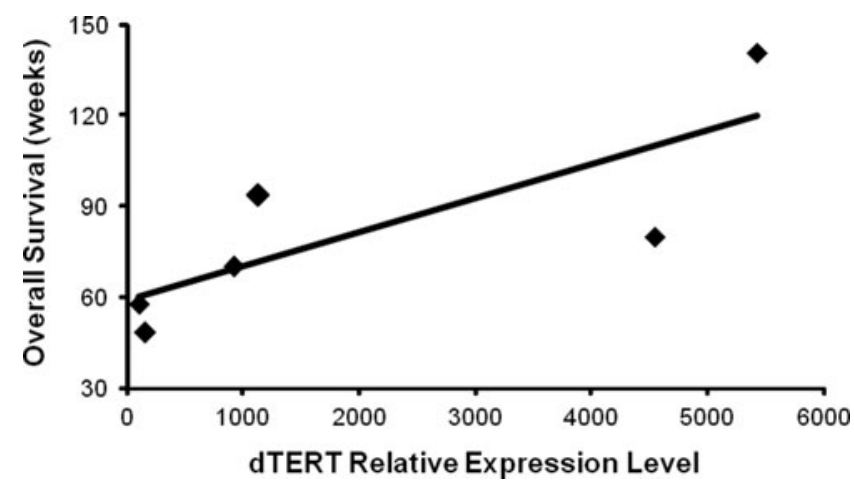

FIG. 4. dTERT is a potential prognostic factor for dTERT vaccinated LSA patients' survival. RNA was extracted from six fine-needle aspirates of lymph nodes from B-cell LSA dogs as described in the text and Materials and Methods. dTERT mRNA was quantified by Taqman real-time polymerase chain reaction. There was a statistically significant association between dTERT expression levels and overall survival among vaccinated patients (Spearman's rho $=0.80, p<0.05$ ). 
immunogenic and most importantly had a significant therapeutic impact on LSA dogs. However, the study was conducted with a relatively limited number of canine patients in comparison with recent cases treated in the same facility by the same veterinarians with the same chemotherapy protocol as the vaccinated patients. Although such a historical control group minimizes the unwanted variability associated with a single-arm study, a double-arm study would increase the confidence in this novel combination therapy. Aiming at this objective, here we have adopted the optimized vaccination schedule consisting of heterologous Ad prime/DNA-EGT boost and run a double-arm study in B-cell LSA dogs in remission treated with standard COP chemotherapy. The primary end point of the study was to assess the efficacy of dTERT vaccination in combination with COP therapy on progression and evolution of canine LSA and overall survival. It has been reported that induction of disease remission by chemotherapy alone in B-cell LSA dogs may by itself be sufficient to generate strong T-cell immunity (Mitchell et al., 2012). Thus, we have first determined whether a spontaneous immune response against dTERT could be detected in healthy dogs and COP-treated LSA patients (Fig. 2A). No significant differences were found, thus suggesting that this chemotherapy protocol does not induce dTERTspecific T lymphocytes. The vaccine was then evaluated in 21 B-cell LSA patients (VAC cohort). The vaccine was immunogenic as shown by a detectable T-cell response against dTERT being noted in about $90 \%$ of the patients, while the response was absent in dogs from the CTR arm (Fig. 2B). In most cases Ad was able to break immune tolerance and DNA-EGT boosted the immune response over time. In addition to its properties as efficient gene delivery vector, the potency of Ad can also explained by the absence of antihuman adenovirus in dogs. Indeed, none of the analyzed vaccinated patients presented pre-existing anti-Ad6 antibodies at priming (data not shown). It would be interesting to measure the pre-existing and induced T-cell immunity against the viral vector. In agreement with our previous observation in healthy dogs (Peruzzi et al., 2010a), the mapping of dTERT immune reactive regions (i.e., containing immunogenic epitopes) showed that the response was patient specific (Fig. 2C) and that in most cases there was more than a single immune dominant portion within the protein component of dog telomerase. This observation reflects the heterogeneity of the population in terms of diverse MHC class I haplotypes and immune system properties. No adverse effects were observed in any dog patient, such as significant changes in hematological parameters (Supplementary Table S1), local/systemic toxicity or organic dysfunction, and fever that could be attributed to the combination treatment, as also reported by the owners. Very importantly, a strong therapeutic impact on survival time was observed in the VAC group over the CTR group (Fig. 3B). In contrast to our previous single-arm study (Peruzzi et al., 2010a), this behavior was not reflected in the progression-free survival outcome (Fig. 3A). We do not have a clear explanation for this difference. Certainly one possibility is that the historical controls of the single-arm study compared with the CTR arm of the current study may show variability due to contingent factors. A second observation is that active immunotherapy needs more time to control the disease and subsequently exert a therapeutic effect. Indeed, cancer im- munotherapy in general acts via a gradual build-up of immune responses in the body that eventually are expected to affect cancer growth and propagation. Cancer vaccine clinical trials in humans indicate that long-term benefits are obtained concurrently with transient tumor growth (Stein et al., 2011). In fact, the dynamics of antitumor effects of immunotherapeutic agents are in general much slower than chemotherapies and patients with a stable disease, or a progressive disease at early time points, may experience tumor regression at a later time. These observations have led to the conclusion that the conventional clinical trial endpoints cannot be applied as such and that new and specific criteria need to be applied for cancer immunotherapy (Hoos et al., 2007; Stein et al., 2011). Another aspect to be considered is that TERT is a particular antigen, as it is expressed, albeit at lower levels, in actively replicating normal cells. Since the goal of the vaccine is the induction and proliferation of TERTspecific T cells, these proliferating TERT-specific T cells could become their own target and delay their action on lymphoma cells. This may explain why there is no difference between vaccinated and control groups regarding progression-free survival. The white blood cell counts would not show this reduction on TERT-specific $\mathrm{T}$ cells because of their low number when compared to the overall white blood cell population. In light of all these considerations, overall survival should be the main and most significant end point to be considered, irrespective of other parameters.

A potential limitation of our study is that we have enrolled and monitored LSA dogs recurrences exclusively on the basis of clinical evaluation (upon physical exam and lymph node palpation plus diagnostic imaging mainly abdominal ultrasonography). Nowadays, novel PCR methods allow the detection of few circulating LSA cells and the establishment of "molecular" rather than the "clinical" remission and recurrence criteria (Burnett et al., 2003; Valli et al., 2006). The standardized use of this technology will certainly allow a timely treatment of patients and a better "fine-tuning" with dTERT immunotherapy. Moreover, another consideration suggesting that there is room for improvement is that COP chemotherapy regimen can be considered almost dated, as other treatment protocols are now accepted in the common practice and do not include chronic chemotherapy (Chun, 2009; Couto, 2009; Lurie et al., 2009; Vail, 2010; see above). It will be of interest to determine how dTERT vaccine could complement these different regimens. We cannot exclude that COP maintenance chemotherapy may act as an crucial immunomodulator in our vaccination protocol. In fact, it is well recognized the synergistic effect of cytotoxic chemotherapy with various immunotherapies in human and veterinary settings (Valli et al., 2006; Bergman, 2007; Sorenmo et al., 2011; O'Connor et al., 2012). Some classes of chemotherapy drugs may affect antigen cross-presentation (van der Most et al., 2006), induce a cytokine storm (Bracci et al., 2007), reduce the number of regulatory T cells (Ghiringhelli et al., 2007), and activate homeostatic lymphoid proliferation (Dudley et al., 2002) that could help inducing the immune response against self antigens (Nistico et al., 2009). The modulation of the activity of T-regulatory cells in dogs with naturally occurring cancer by low-dose chemotherapy (Burton et al., 2011; Mitchell et al., 2012) and the recent identification of canine myeloid suppressor cells (Goulart et al., 2012) definitely provide important tools to better characterize this synergic mechanism of action. 
Finally, the lack of correlation between the dTERT immune response and survival suggests that other surrogate biomarkers of efficacy need to be identified. To identify potential immune correlates, it would be of great interest to further characterize the phenotype of the elicited $T$ cells and assess whether they are able to produce only IFN $\gamma$, or if they are polyfunctional and with cytolytic properties. Our preliminary observation on a limited set of samples suggest that dTERT expression levels are indeed associated with a better patient prognosis (Fig. 4). A possible reason is that dTERT epitopes are more efficiently processed and presented by MHC machinery, thus making the cancer cell more visible to the immune system. We are currently developing a novel immunologic assay aiming at detecting anti-dTERT antibodies in vaccinated patients' serum and are working to characterize the gene expression pattern of LSA patients and identify potential molecular signatures associated with better clinical outcomes, in addition to dTERT expression.

In summary, our double-arm study shows that dTERT vaccine is safe and has a significant impact on LSA canine patients' survival when combined with COP chemotherapy. Our data support the clinical use of dTERT cancer vaccine in LSA and warrant the evaluation of this treatment in other canine tumor types. In this respect, the companion canine represents an informative comparative oncology model regarding clinical benefits for advancement of genetic cancer vaccines to treat human NHL when combined with standard of care chemotherapy.

\section{Acknowledgments}

L.A. work was supported in part by a grant AIRC IG 10507. We thank Cinzia Roffi for editorial assistance and the owners of the dogs for their collaboration and patience as well as the referring practitioners for sending their cases to our attention.

\section{Author Disclosure Statement}

No competing financial interests exist.

\section{References}

Argyle, D.J., and Nasir, L. (2003). Telomerase: a potential diagnostic and therapeutic tool in canine oncology. Vet. Pathol. 40, $1-7$.

Aurisicchio, L., and Ciliberto, G. (2011a). Emerging cancer vaccines: the promise of genetic vectors. Cancers 3, 3687-3713.

Aurisicchio, L., and Ciliberto, G. (2011b). Harnessing the Immune System to Fight Cancer: The Promise of Genetic Cancer Vaccines. Cancer Treatment/Book 1. Intech, Rijeka, Croatia.

Aurisicchio, L., and Ciliberto, G. (2012). Genetic cancer vaccines: current status and perspectives. Expert Opin. Biol. Ther. 12, 1043-1058.

Aurisicchio, L., Mennuni, C., Giannetti, P., et al. (2007). Immunogenicity and safety of a DNA prime/adenovirus boost vaccine against rhesus CEA in nonhuman primates. Int. J. Cancer 120, 2290-2300.

Bergman, P.J. (2007). Anticancer vaccines. Vet. Clin. North Am. Small Anim. Pract. 37, 1111-1119.

Bracci, L., Moschella, F., Sestili, P., et al. (2007). Cyclophosphamide enhances the antitumor efficacy of adoptively transferred immune cells through the induction of cytokine expression, B-cell and T-cell homeostatic proliferation, and specific tumor infiltration. Clin. Cancer Res. 13, 644-653.

Burnett, R.C., Vernau, W., Modiano, J.F., et al. (2003). Diagnosis of canine lymphoid neoplasia using clonal rearrangements of antigen receptor genes. Vet. Pathol. 40, 32-41.

Burton, J.H., Mitchell, L., Thamm, D.H., et al. (2011). Low-dose cyclophosphamide selectively decreases regulatory $\mathrm{T}$ cells and inhibits angiogenesis in dogs with soft tissue sarcoma. J. Vet. Intern. Med. 25, 920-926.

Couto, C.G. (2009). Lymphoma in the cat and dog, In: Small Animal Internal Medicine. 4th edition. R.W. Nelson and C.G. Couto, eds. (Saunders, Elsevier, St. Louis, MO) pp. 1174-1186. Chun, R. (2009). Lymphoma: which chemotherapy protocol and why? Top. Companion Anim. Med. 24, 157-162.

Dharmapuri, S., Peruzzi, D., Mennuni, C., et al. (2009). Coadministration of telomerase genetic vaccine and a novel TLR9 agonist in nonhuman primates. Mol. Ther. 17, 1804-1813.

Dudley, M.E., Wunderlich, J.R., Robbins, P.F., et al. (2002). Cancer regression and autoimmunity in patients after clonal repopulation with antitumor lymphocytes. Science. 298, 850-854.

Fattori, E., Aurisicchio, L., Zampaglione, I., et al. (2009). ErbB2 genetic cancer vaccine in nonhuman primates: relevance of single nucleotide polymorphisms. Hum. Gene Ther. 20, 253265.

Finnefrock, A.C., Tang, A., Li, F., et al. (2009). PD-1 blockade in rhesus macaques: impact on chronic infection and prophylactic vaccination. J. Immunol. 182, 980-987.

Fournel-Fleury, C., Magnol, J.P., Bricaire, P., et al. (1997). Cytohistological and immunological classification of canine malignant lymphomas: comparison with human non-Hodgkin's lymphomas. J. Comp. Pathol. 117, 35-59.

Gavazza, A., Presciuttini, S., Barale, R., et al. (2001). Association between canine malignant lymphoma, living in industrial areas, and use of chemicals by dog owners. J. Vet. Intern. Med. 15, 190-195.

Gavazza, A., Sacchini, F., Lubas, G., et al. (2009). Clinical, laboratory, diagnostic and prognostic aspects of canine lymphoma: a restrospective study. Comp. Clin. Pathol. 18, 291-299.

Ghiringhelli, F., Menard, C., Puig, P.E., et al. (2007). Metronomic cyclophosphamide regimen selectively depletes CD4 + CD25+ regulatory $\mathrm{T}$ cells and restores $\mathrm{T}$ and $\mathrm{NK}$ effector functions in end stage cancer patients. Cancer Immunol. Immunother. 56, 641-648.

Goulart, M.R., Pluhar, G.E., and Ohlfest, J.R. (2012). Identification of myeloid derived suppressor cells in dogs with naturally occurring cancer. PLoS One 7, e33274.

Hayes, H.M., Tarone, R.E., Cantor, K.P., et al. (1991). Casecontrol study of canine malignant lymphoma: positive association with dog owner's use of 2, 4-dichlorophenoxyacetic acid herbicides. J. Natl. Cancer Inst. 83, 1226-1231.

Hoos, A., Parmiani, G., Hege, K., et al. (2007). A clinical development paradigm for cancer vaccines and related biologics. J. Immunother. 30, 1-15.

Lurie, D.M., Gordon, I.K., Théon, A.P., et al. (2009). Sequential low-dose rate half-body irradiation and chemotherapy for the treatment of canine multicentric lymphoma. J. Vet. Intern. Med. 23, 1064-1070.

Marconato, L. (2011). The staging and treatment of multicentric high-grade lymphoma in dogs: a review of recent developments and future prospects. Vet. J. 188, 34-38.

Marconato, L., Gelain, M.E., and Comazzi, S. (2013). The dog as a possible animal model for human non-Hodgkin lymphoma: a review. Hematol. Oncol. 31, 1-9. 
Meyerson, M., Counter, C.M., Eaton, E.N., et al. (1997). hEST2, the putative human telomerase catalytic subunit gene, is upregulated in tumor cells and during immortalization. Cell 90, 785-795

Messaoudi, I., Estep, R., Robinson, B., and Wong, S.W. (2011). Nonhuman primate models of human immunology. Antioxid. Redox Signal. 14, 261-273.

Mitchell, L., Dow, S.W., Slansky, J.E., and Biller, B.J. (2012a). Induction of remission results in spontaneous enhancement of anti-tumor cytotoxic T-lymphocyte activity in dogs with B cell lymphoma. Vet. Immunol. Immunopathol. 145, 597-603.

Mitchell, L., Thamm, D.H., and Biller, B.J. (2012b). Clinical and immunomodulatory effects of toceranib combined with lowdose cyclophosphamide in dogs with cancer. J. Vet. Intern. Med. 26, 355-362.

Nakamura, T.M., Morin, G.B., Chapman, K.B., et al. (1997). Telomerase catalytic subunit homologs from fission yeast and human. Science. 277, 955-959.

Nasir, L. (2008). Telomeres and telomerase: biological and clinical importance in dogs. Vet. J. 175, 155-163.

Nasir, L., Devlin, P., McKevitt, T., et al. (2001). Telomere lengths and telomerase activity in dog tissues: a potential model system to study human telomere and telomerase biology. Neoplasia 3, 351-359.

Nistico, P., Capone, I., Palermo, B., et al. (2009). Chemotherapy enhances vaccine-induced antitumor immunity in melanoma patients. Int. J. Cancer 124, 130-139.

O'Connor, C.M., Sheppard, S., Hartline, C.A., et al. (2012). Adoptive T-cell therapy improves treatment of canine nonHodgkin lymphoma post chemotherapy. Sci. Rep. 2, 249.

Paoloni, M., and Khanna, C. (2008). Translation of new cancer treatments from pet dogs to humans. Nat. Rev. Cancer 8, 147-156.

Peruzzi, D., Gavazza, A., Mesiti, G., et al. (2010). A vaccine targeting telomerase enhances survival of dogs affected by B-cell lymphoma. Mol. Ther. 18, 1559-1567.

Peruzzi, D., Mesiti, G., Ciliberto, G., et al. (2010). Telomerase and HER-2/neu as targets of genetic cancer vaccines in dogs. Vaccine 28, 1201-1208.

Ranieri, G., Gadaleta, C.D., Patruno, R., et al. (2013). A model of study for human cancer: Spontaneous occurring tumors in dogs. Biological features and translation for new anticancer therapies. Crit. Rev. Oncol. Hematol. pii: S10408428(13)00065-6.

Simon, D., Moreno, S.N., Hirschberger, J., et al. (2008). Efficacy of a continuous, multiagent chemotherapeutic protocol versus a short-term single-agent protocol in dogs with lymphoma. J. Am. Vet. Med. Assoc. 232, 879-885.

Sorenmo, K.U., Krick, E., Coughlin, C.M., et al. (2011). CD40activated $\mathrm{B}$ cell cancer vaccine improves second clinical re- mission and survival in privately owned dogs with nonHodgkin's lymphoma. PLoS One 6, e24167.

Staprans, S.I., Feinberg, M.B., Shiver, J.W., and Casimiro, D.R. (2010). Role of nonhuman primates in the evaluation of candidate AIDS vaccines: an industry perspective. Curr. Opin. HIV AIDS 5, 377-385.

Stein, W.D., Gulley, J.L., Schlom, J., et al. (2011). Tumor regression and growth rates determined in five intramural $\mathrm{NCI}$ prostate cancer trials: the growth rate constant as an indicator of therapeutic efficacy. Clin. Cancer Res. 17, 907-917.

Ugel, S., Scarselli, E., Iezzi, M., et al. (2010). Autoimmune B-cell lymphopenia after successful adoptive therapy with telomerasespecific T lymphocytes. Blood 115, 1374-1384.

Vail, D.M. (2010). Hematopoietic tumors-lymphoma. In Textbook of Veterinary Internal Medicine. 7th edition. Vol. 2. S. J. Ettinger and E. C. Feldman, eds. (Saunders, Elsevier, St. Louis, MO) $p p$. 2083-2100.

Valli, V.E., Vernau, W., de Lorimier, L.P., et al. (2006). Canine indolent nodular lymphoma. Vet. Pathol. 43, 241-256.

van der Most, R.G., Currie, A., Robinson, B.W., and Lake, R.A. (2006). Cranking the immunologic engine with chemotherapy: using context to drive tumor antigen cross-presentation towards useful antitumor immunity. Cancer Res. 66, 601-604.

Willmann, M., Mullauer, L., Guija de Arespacochaga, A., et al. (2008). Pax5 immunostaining in paraffin-embedded sections of canine non-Hodgkin lymphoma: A novel canine pan pre-B- and B-cell marker. Vet. Immunol. Immunopathol. 128, 359-365.

Willmann, M., Mullauer, L., Guija de Arespacochaga, A., et al. (2009). Pax5 immunostaining in paraffin-embedded sections of canine non-Hodgkin lymphoma: a novel canine pan pre-B- and B-cell marker. Vet. Immunol. Immunopathol. 128, 359-365.

Yazawa, M., Okuda, M., Setoguchi, A., et al. (1999). Measurement of telomerase activity in dog tumors. J. Vet. Med. Sci. 61, 1125-1129.

Address correspondence to: Dr. Luigi Aurisicchio Takis s.r.l. via di Castel Romano 100 00128 Rome Italy

E-mail: aurisicchio@takis-it.it

Received for publication May 30, 2013;

accepted after revision July 26, 2013.

Published online: July 31, 2013. 